\title{
Calidad en cápsula y enteroscopia
}

\author{
Luis E. Zamora-Nava* \\ Departamento de Endoscopia, Instituto Nacional de Ciencias Médicas y Nutrición Salvador Zubirán, Ciudad de México, México
}

\begin{abstract}
Resumen
Actualmente la calidad en endoscopia se ha generalizado a cada una de las técnicas endoscópicas, tal es el caso de la videocápsula endoscópica (VCE) y enteroscopia. Los principales rubros que se tratan son la adecuada indicación, identificación y manejo adecuado de la patología detectada y complicaciones. Dentro de los principales trabajos que se presentaron en esta DDW son relacionados con la VCE y el empleo de la inteligencia artificial (IA) para el diagnóstico de lesiones vasculares, úlceras, graduación de la preparación intestinal y lectura rápida. También se tocaron puntos relacionados con el incremento en el rendimiento diagnóstico considerando el uso de medicamentos antiplaquetarios, el tiempo en el cual se solicitan los estudios endoscópicos, uso de aditamentos como el Endocuff, así como la prevención de factores asociados a retención gástrica y estudios incompletos.
\end{abstract}

\section{Calidad cápsula y enteroscopia}

La calidad en endoscopia es un concepto necesario y universal, por tal motivo las sociedades y asociaciones de endoscopia de diversas partes del mundo se hicieron a la tarea de desarrollar los estándares mínimos necesarios para poder considerar un procedimiento endoscópico de calidad. Las técnicas de exploración endoluminal del intestino delgado no fueron la excepción y si bien fueron incorporadas recientemente, en la actualidad contamos con los estándares necesarios para poder evaluar la calidad de los estudios de VCE y enteroscopia. En términos generales evalúan el desempeño de los estudios endoscópicos en los dominios mayores y menores (preprocedimiento e intraprocedimiento), siendo los principales la adecuada indicación, identificación y manejo de la patología detectada y complicaciones.

En relación con el dominio preprocedimiento se contempla la indicación, calificación en la calidad de la preparación, así como identificar factores relacionados a estudios incompletos, respecto a este apartado se presentaron los siguientes trabajos:
- Development of a deep learning algorithm to evaluate small bowel preparation quality. En este estudio realizado en Corea con una adecuada metodología se realizó un algoritmo de aprendizaje automático o «profundo" (deep learning) para calificar el grado de preparación intestinal (porcentaje de mucosa visualizada en cada uno de los terciles en que se dividió el intestino delgado), evaluándose 280,000 imágenes en el escenario de prueba y 120,000 en el de validación, además de validarse externamente con 50 estudios de VCE. La exactitud del algoritmo en el escenario de validación externa fue del 93\%, la kappa de acuerdo entre el algoritmo y los médicos fue de 0.672. Cuando se realizó un análisis de sensibilidad el punto de corte para definir preparación adecuada por el programa fue de 2.95 con una área bajo la curva de 0.913 (IC 95\%: 0.83-0.99; $p<0.001)^{1}$.

- Inpatient status is associated with longer transit time and lower completion rate in video capsule endoscopy. En este estudio se encontró que en pacientes hospitalizados la frecuencia de estudios de VCE completos fue menor (75.7 vs. $88.9 \%$; $p<0.001$ ), con 
tiempos de tránsito gástrico e intestinal mayores (35.7 vs. $50.4 \% ; p=0.026$ y 214.1 vs. $274.6 ; p<0.001$, respectivamente), si bien no hubo diferencia en el rendimiento diagnóstico, los pacientes hospitalizados tuvieron mayor frecuencia de hemorragia activa (5.9 vs. $16.9 \%$; $p<0.001$ ), así como mayor necesidad de estudios endoscópicos (13.3 vs. $30.6 \%$; $p<$ 0.001). Finalmente, en pacientes hospitalizados la VCE se asoció a una mayor frecuencia de estudios incompletos (OR: 3.04; IC 95\%: 1.61-5.72; $p<0.001$ ).

- Prolonged gastric time in small-bowel capsule endoscopy which patients are at risk and what implications? En este estudio ( $\mathrm{N}=957)$ se analizaron los factores de riesgo asociados a retardo en el tiempo de tránsito gástrico (RTTG). El RTTG se presentó en el $4.7 \%$ de la población y los fatores asociados fueron la edad ( $52 \pm 20$ vs. $44 \pm 18$ años; $p=0.045$ ), sexo femenino ( 44.2 vs. $18.6 \% ; p=0.01$ ), diabetes (25.6 vs. 9.3\%; $p=0.047$ ), uso de medicamentos psicotrópicos ( 30.2 vs. $11.6 \%, p=0.03$ ), preparación intestinal inadecuada (16.3 vs. $0 \% ; p=0-01)$. De forma importante y a diferencia de lo reportado en otros estudios, el RTTG no se asoció con una mayor frecuencia de estudios incompletos o menor rendimiento diagnóstico lo cual los autores explican que puede relacionarse a que debido a la monitorización del vídeo en tiempo real les permite tomar medidas anticipadas para optimizar el tiempo de exploración de la VCE.

El segundo dominio mayor de los estándares de calidad es la identificación y manejo adecuado de la patología, lo cual va de la mano con el conocer los factores que incrementan el rendimiento diagnóstico como el tiempo en el cual se solicitan los estudios, tipo de presentación de hemorragia (manifiesto/oculto), entre otros. En este congreso se realizaron diferentes trabajos, principalmente en el área de VCE relacionados con el uso de IA para incrementar el rendimiento diagnóstico y reducir tiempos de lectura ${ }^{3}$.

- The new generation of Mirocam Express View is highly accurate and is effective to reduce the capsule endoscopy reading time. Con la finalidad de reducir los tiempos de lectura y optimizar el manejo de los pacientes Mirocam desarrolló un nuevo algoritmo de lectura (Express-View, MC 1200 v.3.0) que elimina imágenes redundantes. En este estudio se incluyeron pacientes diagnósticos de sospecha de hemorragia de intestino delgado y tumores. Se realizó la lectura de los vídeos en modo estándar (MS) y un lector externo realizó la lectura mediante el modo express view (EV), en caso de discrepancia se recurrió a un tercer lector que evaluó los vídeos tanto en modo estándar como EV y fungió como el nuevo estándar de oro. El objetivo primario fue evaluar la sensibilidad de EV para detectar lesiones P1 y P2, el secundario fue la evaluación fue la mediana de tiempo de lectura en MS y EV. En la primera fase existió un acuerdo en la lectura entre ambos sistemas del $85.2 \%$ y una vez que los casos discordantes se evaluaron por un tercer lector, el acuerdo fue del $98.3 \%$ con sensibilidad del $97 \%$, especificidad $100 \%$, VPP $100 \%$ y VPN $95 \%$. En el análisis por lesiones, la sensibilidad fue del $98 \%$, especificidad del $100 \%$, VPP del $100 \%$ y VPN del $93 \%$. El tiempo de lectura fue de 71 minutos (rango: 26-340) para el MS y de 13 minutos (rango: $3-85)$ para el EV $(p<0.001)$ con una reducción en el número de imágenes del $94.8 \%{ }^{4}$. - Automatic detection of enteric ulcers and erosions in capsule endoscopy using a convolutional neural network. Este trabajo evaluó el uso de deep learnig mediante un modelo de redes neuronales convolucionales para la detección automática de erosiones y úlceras, se emplearon 1,483 estudios de VCE (aproximadamente 67,214,009 fotogramas), se seleccionaron 6,130 fotogramas que incluían imágenes normales, erosiones y úlceras (bajo y alto potencial de hemorragia). Las imágenes seleccionadas se sometieron a una doble validación $(80 \%$ para el escenario de entrenamiento y $20 \%$ para el escenario de validación). Se observó que el modelo en la etapa de entrenamiento diagnosticó al $100 \%$ de las úlceras de alto potencial de hemorragia y al $100 \%$ de las imágenes normales, con un mal desempeño en la identificación de las úlceras de bajo potencial de hemorragia y erosiones, lo cual fue mejorando la red neuronal conforme se iba entrenando en la detección de las lesiones, así finalmente se reportó una sensibilidad de $91.7 \%$, especificidad del $97.8 \%$, precisión del $96.7 \%$ y exactitud del $95.9 \%$, el tiempo promedio de procesamiento de solo 23 segundos ${ }^{5}$.

- Endocuff assisted push enteroscopy increases the diagnosis of gastrointestinal angiodysplasia. Los autores de este estudio se plantearon si el uso del Endocuff se asoció con mayores tasas de detección de angiectasias, encontrando que la mayoría de las lesiones se localizaron en el $4-14 \%$ de tiempo de tránsito intestinal y por lo tanto accesibles a enteroscopia de empuje. En los 25 pacientes consecutivos solo en $9(36 \%)$ se detectaron lesiones vasculares cuando se hizo la enteroscopia sin Endocuff comparado con $23(88 \%)$ cuando se empleó, asimismo se observó un incremento acumulado en el número de 
lesiones vasculares de 26 a 112. Los resultados del estudio son interesantes, sin embargo, el diseño no es el mejor, dentro de sus principales limitantes está la falta de aleatorización y cegamiento ${ }^{6}$.

- Antiplatelet therapy at the time of admission predicts positive capsule endoscopy and likelihood of endoscopic intervention. En 311 estudios de VCE con sospecha de hemorragia de intestino delgado en $63 \%$ se documentó consumo de fármacos antiplaquetarios (antiagregantes 50.51\%, anticoagulantes $18.36 \%$, ambos $31.12 \%$ ), los antiagregantes se asociaron con una mayor probabilidad de hallazgos positivos e intervención endoscópica a 90 días (74\%; $p=0.03$ y $36 \% ; p=0.045$, respectivamente). Lo anterior reafirma la recomendación de no suspender el consumo de estos medicamentos durante la realización de los estudios de VCE? .

- (Don't) let the mid gut bleed-impact o enteroscopy. Existe un subgrupo de pacientes con hemorragia manifiesta que se benefician de comenzar con enteroscopia. Este trabajo pone de manifiesto este punto, en un estudio retrospectivo en 751 pacientes consecutivos se realizaron un total de 807 VCE y 407 enteroscopias de doble balón (EDB) observándose un mayor rendimiento diagnóstico en el grupo de EDB comparado con VCE (68.8 vs. $57.7 \%$ ) y principalmente en el subgrupo de EDB en hemorragia activa (75\%). Otro aspecto a considerar en este artículo es que hace referencia a la importancia del tiempo en el que se hacen los estudios y el rendimiento diagnóstico representado por los bajos valores de concordancia a medida que se incrementan los días entre el estudio de VCE y el de EDB ${ }^{8}$.

\section{Financiamiento}

La presente investigación no ha recibido ayudas específicas provenientes de agencias del sector público, sector comercial o entidades sin ánimo de lucro.

\section{Conflicto de intereses}

Los autores declaran que no hay conflicto de intereses.

\section{Bibliografía}

1. Nam JH, Oh DJ, Park J, Song HJ, Lim YJ. ID: 3493047 Development of a deep learning algorithm to evaluate small bowel preparation quality. Gastrointest Endosc [Internet]. 2021;93(6 Suppl):AB351. Disponible en: https://www.giejournal.org/article/S0016-5107(21)01134-2/pdf

2. Bader H, Zamir H, Nehme F, Cheng A-L, Aftab N, Mathews T, et al. ID: 3524782 Inpatient status is associated with longer transit time and lower completion rate in video capsule endoscopy. Gastrointest Endosc [Internet]. 2021;93(6 Suppl):AB351. Disponible en: https://www.giejournal.org/ article/S0016-5107(21)01136-6/fulltext

3. Freitas M, Silva VM, Carvalho PB, Rosa B, Moreira MJ, Cotter J. ID: 3522029 Prolonged gastric transit time in small-bowel capsule endoscopy - which patients are at risk and what implications? Gastrointest Endosc [Internet]. 2021;93(6 Suppl):AB351. Disponible en: https://www.giejournal.org/article/S0016-5107(21)01135-4/abstract

4. Piccirelli S, Mussetto A, Bellumat A, Cannizzaro R, Pennazio M, Pezzoli A, et al. ID: 3525957 The new generation of Mirocam Express View is highly accurate and is effective to reduce the capsule endoscopy reading time. Gastrointest Endosc [Internet]. 2021:93(6 Suppl):AB355. Disponible en: https://www.giejournal.org/article/S0016-5107(21)01143-3/pdf

5. Aoki T, Yamada A, Aoyama K, Saito H, Tsuboi A, Nakada A, et al. Automatic detection of enteric ulcers and erosions in capsule endoscopy using a convolutional neural network. Gastrointest Endosc [Internet] 2021;89(2):P357-363.e2. Disponible en: https://www.giejournal.org/article/S0016-5107\%2818\%2933200-0/fulltext

6. Jackson CS, Kesavan C, Das A, Imbertson E, Strong RM. ID: 3523942 Endocuff assisted push enteroscopy increases the diagnosis of gastrointestinal angiodysplasia. Gastrointest Endosc [Internet]. 2021;93(6 Suppl):AB35. Disponible en: https://www.giejournal.org/article/S00165107(21)00376-X/abstract

7. Boortalary T, Shinn B, Au C, Infantolino A, Tofani CJ. ID: 3524094 Antiplatelet therapy at the time of admission predicts positive capsule endoscopy and likelihood of endoscopic intervention. Gastrointest Endosc [Internet]. 2021;93(6 Suppl):AB354. Disponible en: https://www.giejournal.org/article/S0016-5107(21)01141-X/abstract

8. (Don't) let the mid gut bleed-impact o enteroscopy. 